\title{
Multiscale Complexity Analysis of Short QT Interval Variability Series Stratifies the Arrhythmic Risk of Long QT Syndrome Type 1 Patients
}

\author{
Vlasta Bari ${ }^{1}$, Beatrice De Maria ${ }^{2}$, Giulia Girardengo ${ }^{3}$, Emanuele Vaini ${ }^{1}$, Beatrice Cairo ${ }^{4}$, Lia Crotti ${ }^{3}$, \\ Paul A Brink ${ }^{5}$, Peter J Schwartz ${ }^{3}$, Alberto Porta ${ }^{1,4}$ \\ ${ }^{1}$ Department of Cardiothoracic, Vascular Anesthesia and Intensive Care, IRCCS Policlinico San \\ Donato, San Donato Milanese, Milan, Italy \\ ${ }^{2}$ IRCCS Istituti Clinici Scientifici Maugeri, Istituto di Milano, Milan, Italy \\ ${ }^{3}$ Center for Cardiac Arrhythmias of Genetic Origin, IRCCS Istituto Auxologico Italiano, \\ Centro Diagnostico San Carlo, Milan, Italy \\ ${ }^{4}$ Department of Biomedical Sciences for Health, University of Milan, Milan, Italy \\ ${ }^{5}$ Department of Internal Medicine, University of Stellenbosch, Stellenbosch, South Africa
}

\begin{abstract}
A linear model-based multiscale complexity (MSC) approach was here applied to short heart period (HP) and QT interval variability series derived from 24 hours Holter ECG recordings in a group of long $Q T$ syndrome type 1 (LQT1) patients. The MSC approach allows to assess complexity in the typical frequency bands of $H P$ and $Q T$ variability, i.e. low frequency ( $L F$, from 0.04 to $0.15 \mathrm{~Hz})$ and high frequency $(\mathrm{HF}$, from 0.15 to $0.5 \mathrm{~Hz})$.

MSC was computed along with a single scale complexity over 7 LQT1 asymptomatic mutation carriers (AMC), 22 symptomatic mutation carriers (SMC) and 13 healthy non-mutation carriers (NMC) belonging to the same family line during daytime and nighttime.

Time domain markers and HP variability complexity analyses were unable to separate groups. While single scale QT variability complexity analysis could distinguish NMC from mutation carriers, solely MSC of $Q T$ variability distinguished AMCs from SMCs, showing that AMCs have a reduced complexity in LF band during daytime. We conclude that a reduced complexity of the sympathetic drive directed to the ventricles might be protective against life threatening arrhythmias especially during day being the most risky period for LQT1 patients.

MSC of QT variability could be fruitfully exploited to improve risk stratification in LQT1 population.
\end{abstract}

\section{Introduction}

Long QT syndrome (LQTS) is an inherited disease leading to increased risk for life-threatening arrhythmias, such as torsades de pointes, ventricular fibrillation and sudden death [1]. The main manifestation of the syndrome is the prolongation of the QT interval on the surface electrocardiogram (ECG) due to a delayed repolarization of cardiac myocytes. So far, 12 different mutations leading to LQTS have been identified, being the KCNQ1 mutation the most prevalent one and leading to the so-called LQTS type 1 (LQT1) [2]. Autonomic nervous system plays an important role in defining the arrhythmic risk of LQT1 patients, being at higher risk in situations of emotive and physical stress and during daytime [3,4]. Markers of the autonomic profile, as derived from heart period (HP) and QT interval variability, differentiated asymptomatic and symptomatic mutation carriers (AMCs and SMCs respectively) LQT1 patients [5]. In particular, the assessment of model-free complexity at different time scales was shown helpful for stratifying the arrhythmic risk profile in LQT1 patients $[6,7]$. These model-free multiscale complexity approaches have the main disadvantage of requiring long time series exposing the approach to the influence of nonstationarities, such as slow changes of the mean and variance, known to have a detrimental impact on the computation of complexity markers [8].

Recently, a linear model-based multiscale complexity (MSC) approach has been devised to work over short variability series [9]. In the present study, MSC is originally computed over QT variability. We hypothesize that the application of MSC method to QT interval series could be usefully exploited to assess the different risk profile in LQT1 subjects subdivided in AMCs and SMCs and distinguish them from healthy non-mutation carriers (NMCs) belonging to the same family line [4]. Analysis is carried out during daytime (DAY) and nighttime (NIGHT). 


\section{Methods}

\subsection{Single Scale Complexity Analysis}

The autoregressive (AR) process describes the current variable $Y_{n}$ as a linear combination of $m$ past states plus a random white noise. The coefficients $a_{k}$ with $k=1, \ldots, m$, where $m$ is the model order, were estimated via the Levinson-Durbin recursion from a realization of the AR process $y=\left\{y_{n}, n=1, \ldots, N\right\}$, where $N$ is the series length. The prediction error was computed as the difference between the current value $y_{n}$ and its prediction $\hat{y}_{n}$ assessed on the estimated coefficients $\hat{a}_{k}$. The variance $\hat{\lambda}^{2}$ of the prediction error is an estimate of the variance of the portion of the AR process that remains unpredictable and it is directly linked to the complexity of the AR process [10]. $\hat{\lambda}^{2}$ was referred to as complexity index (CI) in the following. Given that $y$ was normalized to have unit variance, CI ranged from 0 to 1 , where 0 indicates perfect regularity ( $y$ is perfectly predictable) and 1 indicates full irregularity (i.e. $y$ is a white noise realization). The model order is optimized in the range from 8 to 14 via the Akaike information criterion [11].

\subsection{MSC}

The MSC method is grounded on the recent hypothesis that the complexity of the basic components of an AR process can be quantified as the distance of the pole (with positive or null phase) associated to each spectral component [9]. The closer the pole to the unit circle of the complex plane, the sharper the spectral peak, the more regular and less complex the associated oscillations. According to this conjecture, the complexity of the oscillations on a specific frequency band can be computed by averaging the position of the poles with positive or null phase dropping that band and by assessing its distance from the unit circle. This distance was taken as CI based on MSC analysis. The poles were calculated as the roots of the polynomial at the denominator of the transfer function of the AR model [11].

Complexity was calculated in the frequency bands that are traditionally adopted in HP variability analysis, namely low frequency (LF, from 0.04 to $0.15 \mathrm{~Hz}$ ) and high frequency (HF, from 0.15 to $0.5 \mathrm{~Hz}$ ) bands [5]. The transformation of the phase of the poles into frequencies was obtained by dividing the normalized phase $\varphi / 2 \pi$ of the pole by the HP mean [12].

According to the frequency band in which $\mathrm{CI}$ is calculated, it is termed as $\mathrm{CI}_{\mathrm{LF}}$ and $\mathrm{CI}_{\mathrm{HF}}$. $\mathrm{CI}_{\mathrm{LF}}$ and $\mathrm{CI}_{\mathrm{HF}}$ range from 0 to 1 , where 0 indicate perfectly regularity and 1 maximum complexity.

\section{Experimental Protocol and Analysis}

Forty-two 12 lead 24 hours Holter ECG recordings (Mortara Instrument Inc., Milwaukee, WI, USA) were acquired from 13 NMCs (age: $38 \pm 11$ years, mean \pm standard deviation, 6 males), 7 AMCs (age: $42 \pm 12$ years, 2 males) and 22 SMCs (age: $37 \pm 15$ years, 8 males). The groups were not different for age and gender. All AMCs and SMCs were mutation carriers (MCs) and carried the same $K C N Q 1$ A $341 \mathrm{~V}$ mutation leading to LQT1. Both MCs and NMCs were member of the same founder population as described earlier [4]. The study was performed in keeping with the Helsinki declaration for studies involving human subjects. The universities of Stellenbosh and Pavia local ethical committees approved the protocol. All subjects signed an informed consent before participating in the study. ECG sampling rate was $180 \mathrm{~Hz}$. Analyses were carried out over the ECG lead with the best signal-to-noise ratio. HP and QT time series were extracted by approximating HP as the time distance between two consecutive R-wave peaks and QT as the time interval between the second $\mathrm{R}$-wave peak delimiting the HP and the end of the T-wave detected with a method based on thresholding the first derivative calculated over the T-wave downslope. Series were manually checked for misdetections or missing beats. A maximum of $5 \%$ of corrections for each series was allowed. Epochs with a length of 5000 consecutive HP and QT values were randomly extracted during DAY (from 2:00 to 7:00 PM) and NIGHT (from 12:00 to 4:00 AM).

Mean and variance of HP and QT series, indicated as $\mu_{\mathrm{HP}}, \mu_{\mathrm{QT}}, \sigma^{2} \mathrm{HP}$ and $\sigma^{2} \mathrm{QT}$ and expressed respectively in ms, $\mathrm{ms}, \mathrm{ms}^{2}$ and $\mathrm{ms}^{2}$, and complexity indexes were assessed on segments of HP and QT series with length equal to 300 cardiac beats and with an overlap of 100 cardiac beats during DAY and NIGHT. The median of the distribution of each index over the entire period was taken as representative of the overall distribution and utilized for any successive statistical analysis. Markers of complexity were indicated as $\mathrm{CI}_{\mathrm{HP}}, \mathrm{CI}_{\mathrm{QT}}, \mathrm{CI}_{\mathrm{LF}, \mathrm{HP}}, \mathrm{CI}_{\mathrm{LF}, \mathrm{QT}}$, $\mathrm{CI}_{\mathrm{HF}, \mathrm{HP}}$, and $\mathrm{CI}_{\mathrm{HF}, \mathrm{QT}}$.

Two-way repeated measures analysis of variance (one factor repetition) was applied to assess statistical differences between NMCs, AMCs and SMCs during DAY and NIGHT. Holm-Sidak test was used for post hoc multiple comparisons. A $p<0.05$ was always deemed as significant.

\section{Results}

Table 1 shows time domain indexes derived from HP and QT series in NMCs, AMCs and SMCs. $\mu_{\mathrm{HP}}$ and $\mu_{\mathrm{QT}}$ lengthened significantly during NIGHT with respect to DAY in all groups with $\mu_{\mathrm{QT}}$ resulting longer in AMCs and SMCs versus NMCs during both DAY and NIGHT. In 
Table 1. Time domain indexes from HP and QT series in NMCs, AMCs and SMCs during DAY and NIGHT.

\begin{tabular}{c|ccc|ccc}
\hline \multirow{2}{*}{ Parameter } & \multicolumn{3}{|c|}{ DAY } & \multicolumn{3}{c}{ NIGHT } \\
\cline { 2 - 6 } & NMC & AMC & SMC & NMC & AMC & SMC \\
\hline$\mu_{\mathrm{HP}}[\mathrm{ms}]$ & $724 \pm 149$ & $760 \pm 129$ & $747 \pm 86$ & $872 \pm 126^{*}$ & $953 \pm 138^{*}$ & $942 \pm 111^{*}$ \\
$\sigma^{2} \mathrm{HP}\left[\mathrm{ms}{ }^{2}\right]$ & $1509 \pm 1440$ & $1796 \pm 1379$ & $1568 \pm 1537$ & $2039 \pm 1771$ & $1602 \pm 756$ & $2449 \pm 2302^{*}$ \\
$\mu_{\mathrm{QT}}[\mathrm{ms}]$ & $321 \pm 45$ & $396 \pm 46^{\S}$ & $402 \pm 43^{\S}$ & $362 \pm 34^{*}$ & $427 \pm 42^{*}$ & $442 \pm 28^{*}$ \\
$\sigma^{2} \mathrm{QT}\left[\mathrm{ms}^{2}\right]$ & $217 \pm 340$ & $217 \pm 194$ & $93 \pm 75$ & $84 \pm 77^{*}$ & $81 \pm 71^{*}$ & $88 \pm 56$ \\
\hline
\end{tabular}

$\mu_{\mathrm{HP}}=\mathrm{HP}$ mean; $\sigma^{2} \mathrm{HP}=\mathrm{HP}$ variance; $\mu_{\mathrm{QT}}=\mathrm{QT}$ mean; $\sigma^{2} \mathrm{QT}=\mathrm{QT}$ variance; NMC=non-mutation carrier; $\mathrm{AMC}=$ asymptomatic mutation carrier; SMC=symptomatic mutation carrier; DAY=daytime; NIGHT=nighttime. Results are reported as mean \pm standard deviation. The symbols * and $\S$ indicate $p<0.05$ versus DAY and versus NMC respectively.

SMCs $\sigma^{2} \mathrm{HP}$ significantly increased during NIGHT compared to DAY, while it did not differ between conditions in NMCs and AMCs or between groups. $\sigma^{2} \mathrm{QT}$ during DAY was larger than during NIGHT in NMCs and AMCs.

Figure 1 shows results of complexity assessed as CI (Fig.1a,b), $\mathrm{CI}_{\mathrm{LF}}$ (Fig.1c,d) and $\mathrm{CI}_{\mathrm{HF}}$ (Fig.1e,f) on $\mathrm{HP}$ (Fig.1a,c,e) and QT series (Fig.1b,d,f). Grouped bar graphs are presented for NMC (grey bars), AMC (white bars) and SMC (black bars) subjects as a function of the experimental condition (i.e. DAY and NIGHT). $\mathrm{CI}_{\mathrm{HP}}$ increased significantly during NIGHT in SMCs. $\mathrm{CI}_{\mathrm{LF}, \mathrm{HP}}$ and $\mathrm{CI}_{\mathrm{HF}, \mathrm{HP}}$ did not differ between groups or conditions. At difference with $\mathrm{HP}$ complexity analysis, $\mathrm{CI}_{\mathrm{QT}}$ was able to differentiate groups, being larger in NMCs with respect to AMCs and SMCs during both DAY and NIGHT. $\mathrm{CI}_{\mathrm{LF}, \mathrm{QT}}$ in AMCs during DAY was smaller than in NMCs and SMCs and within the same group increased during NIGHT. $\mathrm{CI}_{\mathrm{HF} \text {,QT }}$ significantly increased during NIGHT in all groups.

\section{Discussion}

The main findings of this work are: i) MSC of QT interval variability carries complementary information with respect to single scale complexity analysis; ii) MSC of QT variability was able to differentiate LQT1 AMCs and SMCs; iii) LQT1 AMCs have a lower complexity directed to the ventricles in LF band compared to NMCs and SMCs.

Time domain results confirmed the expected circadian behavior of subjects and the main phenotype of the pathology, allowing to separate NMCs from MCs but resulting unable to differentiate AMCs from SMCs.

Short-term HP complexity was confirmed to be under vagal control in LQT1 subjects, being increased during NIGHT especially in SMCs [6,7]. On the contrary, model-based single scale complexity of QT interval variability was unmodified during NIGHT, but differed between groups, resulting larger in NMCs than in MCs. However, this single scale analysis was unable to distinguish SMCs from AMCs. Remarkably, only MSC of QT interval variability in LF band was able to separate different phenotypes, i.e. AMCs from SMCs, while the same method applied on HP could not. As a matter of fact, $\mathrm{CI}_{\mathrm{LF}, \mathrm{QT}}$ in AMCs during DAY was smaller than in NMCs and SMCs and with respect to NIGHT. Since QT variability in LF band is known to be related to the sympathetic modulation directed to the ventricles [13], these results suggest that having a smaller complexity of the sympathetic control directed to the ventricles would be protective in LQT1 patients. This finding confirms our previous studies [6,7]. Since MSC approach exploited shorter sequences than model-free multiscale complexity utilized in [6,7], this result suggests, on the one hand, that nonstationarities did not play a relevant role in the
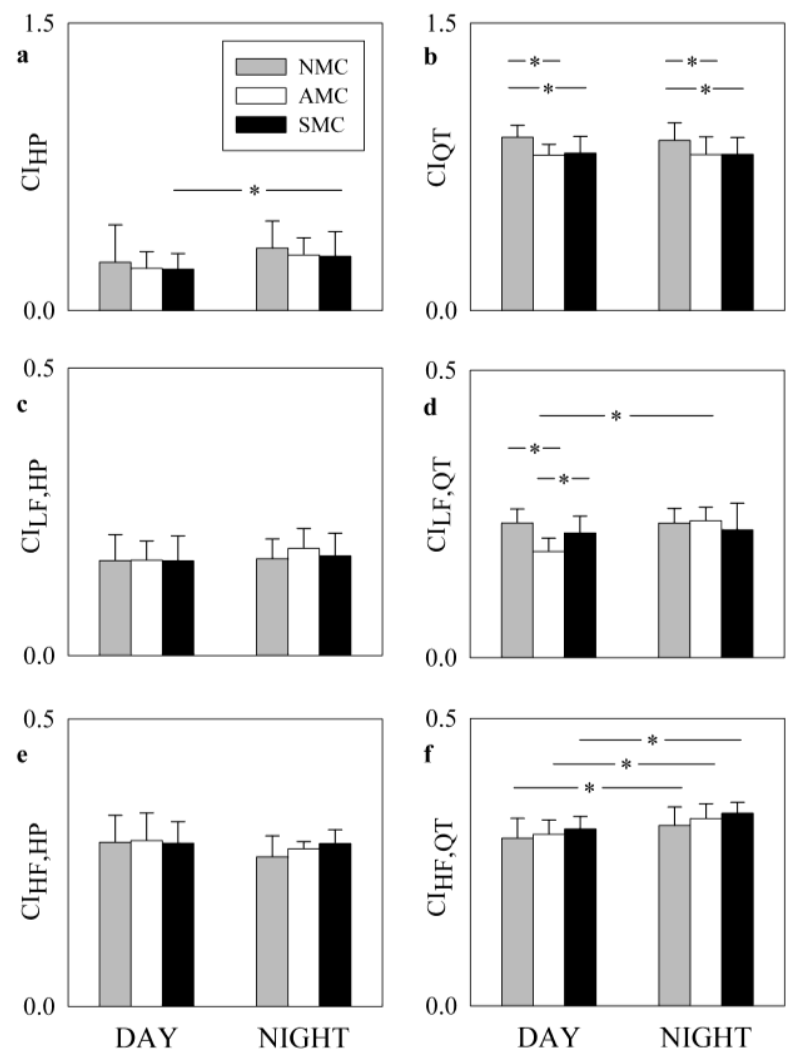

Figure 1. Grouped error bar graphs show CI $(a, b), C_{L F}$ $(\mathrm{c}, \mathrm{d}), \mathrm{CI}_{\mathrm{HF}}(\mathrm{e}, \mathrm{f})$ calculated over HP (a,c,e) and QT (b,d,f) in NMC (grey bars), AMC (white bars) and SMC (black bars) subjects as a function of experimental condition (i.e. DAY and NIGHT). The symbol * indicates a significant difference with $p<0.05$. 
conclusions drawn in $[6,7]$, and, on the other hand, that short-term complexity analysis can be exploited in analyzing 24 hours Holter QT variability recordings. Furthermore, this differentiation was present only during the period at highest arrhythmic risk in LQT1 subjects (i.e. the DAY) [4]. Remarkably, $\mathrm{CI}_{\mathrm{LF}, \mathrm{QT}}$ of $\mathrm{NMCs}$ and SMCs were similar, thus suggesting that AMCs has a peculiar protective feature.

On the contrary, $\mathrm{CI}_{\mathrm{HF}, \mathrm{QT}}$ did not differ between groups but it was increased in all the populations during NIGHT. Since QT interval is mainly under sympathetic control, this finding could be related to the higher level of broad band noise superimposed on QT interval variability series resulting from the low time resolution of this historical database. We advocate further confirmation of this observation by applying the same approach to QT variability extracted from 24 hours Holter ECG recordings obtained via state-of-the-art acquisition technologies.

\section{Conclusions}

Short-term MSC of QT variability seems to be helpful in stratifying the arrhythmic risk in LQT1 patients because it was able to distinguish AMCs from SMCs. This separation occurs in a band that it is likely to be under sympathetic control (i.e. the LF band) and during the most risky period for this population (i.e. the DAY). Neither time domain HP and QT indexes, nor single scale complexity analysis was able to achieve the same conclusions. MSC markers of QT variability deserve to be tested in a more complete model of risk to check whether they could provide complementary information to more traditional indicators of risk in LQT1 population.

\section{References}

[1] P. J. Schwartz, S. G. Priori, C. Spazzolini, A. J. Moss, G. M. Vincent, C. Napolitano, I. Denjoy, P. Guicheney, G. Breithardt, M. T. Keating, J. A. Towbin, A. H. Beggs, P. Brink, A. A. Wilde, L. Toivonen, W. Zareba, J. L. Robinson, K. W. Timothy, V. Corfield, D. Wattanasirichaigoon, C. Corbett, W. Haverkamp, E. Schulze-Bahr, M. H. Lehmann, K. Schwartz, P. Coumel and R. Bloise, "Genotype-phenotype correlation in the long-QT syndrome: gene-specific triggers for lifethreatening arrhythmias," Circulation, vol. 103, pp. 89-95, 2001.

[2] L. Crotti, C. Spazzolini, P. J. Schwartz, W. Shimizu, I. Denjoy, E. Schulze-Bahr, E. V. Zaklyazminskaya, H. Swan, M. J. Ackerman, A. J. Moss, A. A. Wilde, M. Horie, P. A. Brink, R. Insolia, G. M. De Ferrari and G. Crimi, "The common long-QT syndrome mutation KCNQ1/A341V causes unusually severe clinical manifestations in patients with different ethnic backgrounds: toward a mutation-specific risk stratification," Circulation, vol. 116, pp. 2366-2375, 2007.

[3] P. J. Schwartz, "Sudden cardiac death, founder populations, and mushrooms: what is the link with gold mines and modifier genes?" Heart Rhythm, vol. 8, pp. 548-550, 2011.

[4] P. A. Brink, L. Crotti, V. Corfield, A. Goosen, G. Durrheim, P. Hedley, M. Heradien, G. Geldenhuys, E. Vanoli, S. Bacchini, C. Spazzolini, A. L. Lundquist, D. M. Roden, A. L. George Jr and P. J. Schwartz, "Phenotypic variability and unusual clinical severity of congenital longQT syndrome in a founder population," Circulation, vol. 112, pp. 2602-2610, 2005.

[5] A. Porta, G. Girardengo, V. Bari, A. L. George Jr, P. A. Brink, A. Goosen, L. Crotti and P. J. Schwartz, "Autonomic control of heart rate and QT interval variability influences arrhythmic risk in long QT syndrome type 1," J. Am. Coll. Cardiol., vol. 65, pp. 367-374, 2015.

[6] V. Bari, J. F. Valencia, M. Vallverdu, G. Girardengo, A. Marchi, T. Bassani, P. Caminal, S. Cerutti, A. L. George Jr, P. A. Brink, L. Crotti, P. J. Schwartz and A. Porta, "Multiscale complexity analysis of the cardiac control identifies asymptomatic and symptomatic patients in long QT syndrome type 1," PLoS ONE, vol. 9, art. no. e93808, 2014.

[7] V. Bari, A. Marchi, B. De Maria, G. Girardengo, A. L. J. George, P. A. Brink, S. Cerutti, L. Crotti, P. J. Schwartz and A. Porta, "Low-pass filtering approach via Empirical Mode Decomposition improves short scale entropy-based complexity estimation of QT interval variability in Long QT Syndrome Type 1 Patients," Entropy, vol. 16, pp. 48394854, 2014.

[8] V. Magagnin, T. Bassani, V. Bari, M. Turiel, R. Maestri, G.D. Pinna and A. Porta, "Non-stationarities significantly distort short-term spectral, symbolic and entropy heart rate variability indexes," Physiol. Meas., vol. 32, pp. 1775 1786, 2011.

[9] A. Porta, V. Bari, G. Ranuzzi, B. De Maria and G. Baselli, "Assessing multiscale complexity of short heart rate variability series through a model-based linear approach," Chaos, vol. 27, art. no. 093901, 2017.

[10] A. Porta, B. De Maria, V. Bari, A. Marchi and L. Faes, "Are nonlinear model-free conditional entropy approaches for the assessment of cardiac control complexity superior to the linear model-based one?," IEEE Trans. Biomed. Eng., vol. 64, pp. 1287-1296, 2017.

[11] G. Baselli, A. Porta, O. Rimoldi, M. Pagani and S. Cerutti, "Spectral decomposition in multichannel recordings based on multivariate parametric identification," IEEE Trans. Biomed. Eng., vol. 44, pp. 1092-1101, 1997.

[12] R. W. de Boer, J. M. Karemaker and J. Strackee, "Spectrum of a series of point events, generated by the integral pulse frequency modulation model," Med. Biol. Eng. Comput., vol. 23, pp. 138-142, 1985.

[13] A. Porta, V. Bari, F. Badilini, E. Tobaldini, T. GnecchiRuscone and N. Montano, "Frequency domain assessment of the coupling strength between ventricular repolarization duration and heart period during graded head-up tilt," J. Electrocardiol., vol. 44, pp. 662-668, 2011.

Address for correspondence.

Dr Vlasta Bari, PhD

IRCCS Policlinico San Donato - Department of Cardiothoracic, Vascular Anesthesia and Intensive Care

Via F.Fellini 4, 20097 San Donato Milanese, Milan, Italy

E-mail: vlasta.bari@grupposandonato.it 